\title{
Attributes Underlying Non-surgical Treatment Choice for People With Low Back Pain: A Systematic Mixed Studies Review
}

\author{
Thomas G. Poder ${ }^{1,2^{*}}{ }^{\mathbb{D}}$, Marion Beffarat ${ }^{3}$
}

\begin{abstract}
Background: The knowledge of patients' preferences in the medical decision-making process is gaining in importance. In this article we aimed to provide an overview on the importance of attributes underlying the choice of non-surgical treatments in people with low back pain (LBP).

Methods: A systematic mixed studies review was conducted. Articles were retrieved from the search engines PubMed, ScienceDirect, and Scopus through June 21, 2018. The Mixed Methods Appraisal Tool (MMAT) was used to assess the quality of the study, and each step was performed by 2 reviewers.

Analysis: From a total of 390 articles, 13 were included in the systematic review, all of which were considered to be of good quality. Up to 40 attributes were found in studies using various methods. Effectiveness, ie, pain reduction, was the most important attribute considered by patients in their choice of treatment. This attribute was cited by 7 studies and was systematically ranked first or second in each. Other important attributes included the capacity to realize daily life activities, fit to patient's life, and the credibility of the treatment, among others.

Discussion: Pain reduction was the most important attribute underlying patients' choice for treatment. However, this was not the only trait, and future research is needed to determine the relative importance of the attributes.

Keywords: Low Back Pain, Preference, Treatment, Choice, Systematic Review

Copyright: () 2021 The Author(s); Published by Kerman University of Medical Sciences. This is an open-access article distributed under the terms of the Creative Commons Attribution License (http://creativecommons.org/licenses/ by/4.0), which permits unrestricted use, distribution, and reproduction in any medium, provided the original work is properly cited.

Citation: Poder TG, Beffarat M. Attributes underlying non-surgical treatment choice for people with low back pain: a systematic mixed studies review. Int J Health Policy Manag. 2021;10(4):201-210. doi:10.34172/ijhpm.2020.49
\end{abstract}

Article History:

Received: 11 August 2019 Accepted: 30 March 2020 ePublished: 8 April 2020

\section{Introduction}

Low back pain (LBP) is a common condition experienced by most individuals at least once during their lifetime. ${ }^{1,2}$ LBP refers to pain located between the lower rib margins and the buttock creases. ${ }^{3}$ Generally, the lower back is where most back pain occurs. According to the National Institute of Neurological Disorders and Strokes, ${ }^{4}$ a branch of the National Institute of Health, chronic LBP is defined "as pain that persists for 12 weeks or longer."

In industrialized countries, the prevalence of LBP in a person's lifetime was assessed at $60 \%$ to $70 \%{ }^{5}$ and the incidence rate was between $60 \%$ and $90 \% .^{6}$ An evolution toward chronicity of LBP was observed in 6 to $8 \%$ of cases. ${ }^{7,8}$ Throughout the world, chronic LBP has high economic/ professional (incapacity, absenteeism, activity limitation) and social (isolation, decrease in quality of life, constant need of care) impact on the population. Indeed, chronic LBP is the second cause of incapacity after cardiovascular disease. ${ }^{9}$ To effectively treat this population is essential. However, to be effective, these treatments must adhere to patients' concerns, values and beliefs, and thus, consider their preferences. ${ }^{10}$

According to Bowling and Ebrahim, ${ }^{11}$ treatment preference is defined as the option chosen by the patient after having assessed the risks and benefits of available actions. To take into account the preference of patients in their choice of treatment is especially important in LBP, considering the large number of potential treatments, ie, more than 200 according to Haldeman and Dagenais, ${ }^{12}$ and their relatively low effectiveness. ${ }^{13}$ In addition, Aboagye ${ }^{14}$ puts forward other reasons for which preferences need to be examined in the treatment of this specific condition, including patient empowerment and satisfaction.

According to the Common Sense Model, ${ }^{15}$ a widely used theoretical framework to explain the processes by which patients become aware of and interact with a health threat, patients develop treatment preferences when attempting to match their illness representations with treatment beliefs. Therefore, it is important to consider what drives their choice for treatment and to better understand their preferences for the various attributes (ie, characteristics) describing a given treatment. This is also highlighted by Aboagye ${ }^{14}$ and the National Institute for Health and Care Excellence, ${ }^{16}$ who indicate that preferences and individual values are important and must be considered in the intervention choice process.

To contribute to a better understanding of which preferences drive treatment choice in LBP patients, we conducted a systematic mixed studies review. Specifically, the purpose of this article is twofold: (1) to determine which non- 
surgical treatment attributes are important for patients in their decision-making process, and (2) to report the ranking of these attributes in order of patients' preferences.

\section{Methods}

A systematic mixed studies review of the literature was conducted on non-surgical treatment preferences of people with LBP. To do so, we followed the statement rules used in our health technology assessment unit (unpublished), which are very close to what is described in the guideline developed for systematic reviews by the Institut national d'excellence en santé et en services sociaux (INESSS), ${ }^{17}$ the national health technology assessment agency in Quebec, Canada. The rational for a systematic mixed studies review was to get as much information as possible on this specific topic which may have been understudied. In addition, studying attributes that drive non-surgical treatment preferences will help decision-makers in our institution to reorganize the patients' trajectory of care and to offer patients alternatives to surgical care. The methodological quality of each study was evaluated using the Mixed Methods Appraisal Tool (MMAT)..$^{18}$ In our review protocol, the inclusion criteria were established so as to be as exhaustive as possible. These criteria included studies analyzing health preferences regardless of the method used, eg, discrete choice experiment (DCE), qualitative studies, mix method design, ranking studies, swing weighting studies, analytical hierarchy process, and best-worst scaling. We also used studies referring to acute or chronic pain treatments in the low back region. Exclusion criteria were: preferences other than those of patients, sub-studies of other studies, studies about utilities associated with any health condition, studies combining data from patients with pain other than in the low back region, and studies that only referred to surgical treatment (ie, a study could compare surgical treatment with non-surgical treatment, but could not compare two surgical treatments). There was no limitation of language.

As per protocol, inclusion and exclusion criteria were established before conducting searches in the electronic database and were applied to the final search field. The search engines used in this systematic review were PubMed, ScienceDirect, and Scopus. In addition, to consider unpublished studies we completed the review by scanning references of included studies and contacted the authors who had performed a literature review prior to conducting their research. However, we did not perform a specific search in the grey literature. The search was conducted without date limits through June 21, 2018, using combinations of key search terms such as: "low back pain," "lumbosacral region," "health preference," "patient preference," "stated preference," "stated choice," and "treatment." The complete search strategy based on keywords is available in Supplementary file 1.

Two reviewers (TGP and MB) independently screened the titles and abstracts (first phase of selection) using the criteria. If the criteria were met, the article was selected for a full reading (second phase of selection). The complete readings as well as the scoring with the MMAT were carried out by the 2 independent reviewers. After a full reading, articles were included if they corresponded to inclusion and exclusion criteria. At each step, disagreements were solved with an arbitration performed by a third reviewer. For both phases of selection, Cohen's kappa coefficients were calculated to measure the degree of agreement. The value of the coefficients can be interpreted as follows: values $\leq 0$ indicated no agreement; $0.01-0.20$, none to slight; $0.21-0.40$, fair; $0.41-$ 0.60 , moderate; $0.61-0.80$, substantial; and $0.81-1.00$ was almost perfect agreement. Data were extracted by 1 reviewer (MB) and a second reviewer (TGP) checked and completed this data for accuracy. Any additional information added in the extraction grid was discussed between the 2 reviewers and disagreements were solved by the arbitration of a third reviewer. The main variables of interest in this systematic review were the preferences attributes and their levels. The following variables were also systematically collected: country, type of study, type of treatment, numbers of patients and their characteristics, results as a ranking or a size effect, type of statistical analysis, and other available characteristics, such as the recruitment process and the nature of the treatment experienced. Authors were contacted when data could not be retrieved from the selected articles. The data collected were examined and found to be inappropriate for a meta-analysis considering the high heterogeneity in the study designs and results (ie, different methods to assess preferences, different choice and definition of attributes and levels, different ways to report results). The relative importance of attributes was reported according to the ranking provided by the authors of the included studies.

\section{Results}

In total, 390 studies were identified after the removal of duplicates, 37 of which were fully read to assess their eligibility. A total of 13 studies were selected to be included in the systematic mixed studies review. The Cohen's kappa coefficient was 0.7937 in the first phase of the selection process (screening of both titles and abstracts) and 0.9217 in the second phase (full-text readings). The reasons for excluding 24 studies that were fully read were as follows: the study was a systematic review without original data $(n=3)^{19-21}$; the study did not consider the preferences of patients $(n=4)^{13,14,22,23}$; the study analyzed preferences but not for treatment characteristics $(n=11)^{24-34}$; the pain site was somewhere other than in the low back or data were aggregated with other sites $(\mathrm{n}=4)^{35-38}$; the study was a sub-study of another one $(\mathrm{n}=1)^{39}$; and data was not available even after contacting the authors $(n=1)^{40}$. Details of the process selection can be found in the Preferred Reporting Items for Systematic Reviews and MetaAnalyses (PRISMA) flow diagram in Figure.

Table 1 lists the 13 selected studies. ${ }^{41-53}$ A majority of these studies $(n=7)$ were published during the past 5 years and mainly originated from Europe $(n=7)$ and the United States $(n=3)$. This shows that the topic of health preferences is increasingly gaining importance in the Western world. Very few information about the characteristics of the respondents were provided in the studies, with the exception of age and gender. Of the 11 studies that reported these data, mean age ranged from 41 to 62 years, and mean proportion of women was between $50.4 \%$ and $75.6 \%$. Seven of the included studies 


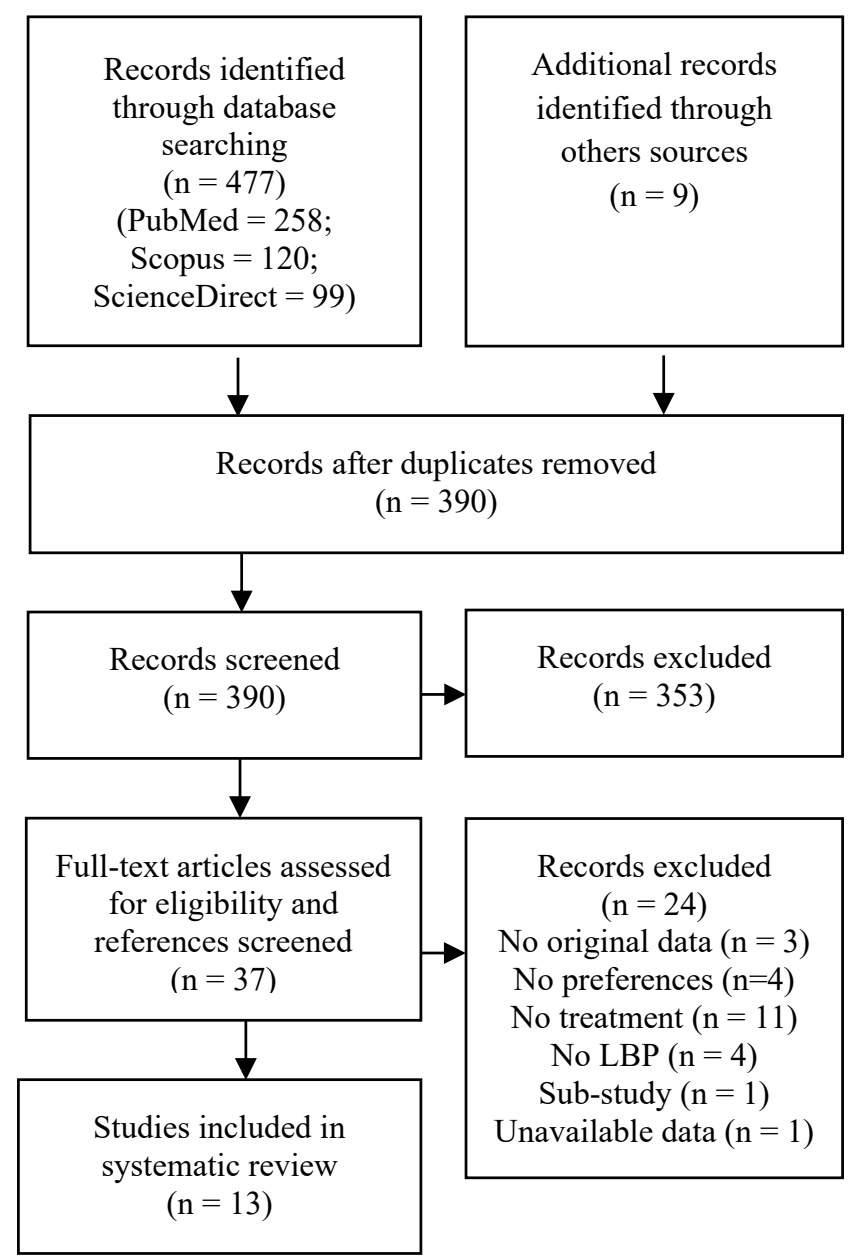

Figure. PRISMA Flow Diagram. Abbreviations: PRISMA, Preferred Reporting Items for Systematic Reviews and Meta-Analyses; LBP, low back pain. were qualitative, while the others were mixed-method or quantitative studies, including 4 DCEs. In general, included studies had a satisfactory score of quality. None of these studies had a score below 50\% in the MMAT. In addition, studies with lower scores were mainly because of missing information in their method's section. As a result, the MMAT score had little impact on how to interpret the findings. A very high heterogeneity in study designs was observed in this systematic review. In particular, the primary studies each used specific measurement methods for patients' preferences. Some were measured with questionnaires and others used focus groups or individual interviews, while the DCE studies used different attributes and levels for treatments. This precluded performing a meta-analysis.

Results of the systematic mixed studies review are reported in Table 2. According to studies included in this review, the attributes most frequently cited in the preferences of patients were effectiveness (ie, reduction in pain level), the capacity to realize daily life activities, fit to the patient's life, providers' attitudes and characteristics, and the frame/design of the treatment (eg, supervised or not, in groups or individually). These attributes were cited in at least four studies. Among these five attributes, effectiveness and capacity to realize daily life activities appeared to be the most valued, while providers' attitudes and characteristics seemed to be much less important.

Alternatively, other attributes were less frequently cited but revealed strong preferences. This was particularly the case for credibility of treatment, capacity to return to work, and treatment frequency. These three attributes were cited in three studies each. Other attributes were also cited in three studies, but revealed less important preferences: onset of

Table 1. Characteristics of Studies Included in the Systematic Review

\begin{tabular}{|c|c|c|c|c|c|}
\hline Authors/Year & Country & Study's Method & No. of Patients & MMAT Score & Source of Funding \\
\hline François et al/2018 & USA & Quantitative (cross-section) & 104 & $68.75 \%$ & NIHR, NICHD, NCMRR \\
\hline Aboagye et al/2017 & Sweden & Quantitative (DCE) & 112 & $95.85 \%$ & $\begin{array}{l}\text { AFA Insurance, Swedish Research Council for Health, } \\
\text { Working Life and Welfare }\end{array}$ \\
\hline Verbrugghe et al/2017 & Belgium & Mixed method (interviews questionnaires) & 40 & $58 \%$ & Not declared \\
\hline Chen et al/2015 & China & Quantitative (DCE) & 86 & $75 \%$ & Research Committee of the University of Macau \\
\hline Dima et al/2015 & England & Quantitative (questionnaires) & 115 & $70.5 \%$ & NIHR School for Primary Care Research \\
\hline Gardner et al/2015 & Australia & Qualitative (Interviews) & 20 & $70.83 \%$ & Self-financing \\
\hline Klojgaard et al/2014 & Denmark & Quantitative (DCE) & 348 & $83.35 \%$ & Danish Strategic Research Council project CeSpine \\
\hline Dima et al/2013 & England & Qualitative (focus group) & 75 & $81.25 \%$ & NIHR School for Primary Care Research \\
\hline Haanstra et al/2013 & USA & Qualitative (interviews) & 77 & $77.1 \%$ & Not declared \\
\hline Klojgaard et al/2012 & Denmark & Qualitative (interviews) & 3 & $91.65 \%$ & Danish Strategic Research Council project CeSpine \\
\hline Yi et al/2011 & Scotland & Quantitative study (DCE) & 124 & $62.5 \%$ & $\begin{array}{l}\text { Scottish Government Health Directorate and Aberdeen } \\
\text { University }\end{array}$ \\
\hline Hsu et al/2010 & USA & Qualitative (interviews) & 327 & $64.62 \%$ & NIH-NCCAM, NIAMSD \\
\hline Slade et al/2009 & Australia & Qualitative (focus group) & 18 & $58.35 \%$ & $\begin{array}{l}\text { National Health and Medical Research Council PhD } \\
\text { Scholarship }\end{array}$ \\
\hline
\end{tabular}

Abbreviations: MMAT, Mixed Methods Appraisal Tool - the score provided is the mean of both reviewers; DCE, discrete choice experiment; NIHR, National Institute for Health Research; NICHD, National Institute of Child Health and Human Development; NCMRR, National Center for Medical Rehabilitation Research; NIH, National Institute for Health; NCCAM, National Center for Complementary and Alternative Medicine; NIAMSD, National Institute for Arthritis and Musculoskeletal and Skin Disease. 
Table 2. Preferences of Patients for Each Attribute of Treatments

\begin{tabular}{|c|c|c|c|}
\hline Attribute & Importance/Ranking & Treatment Modality (Levels) & Reference/Year \\
\hline Effectiveness/pain reduction & $\begin{array}{l}\text { Relevant (determined during focus group) Same weight but prioritised by patients, top } 4 \\
\text { Relevant (determined during focus group) Same weight but prioritised by authors, top } 4 \\
\text { Relevant (validated questionnaire) Same weight - ranked } 2-4 \text { over } 4 \text { attributes } \\
\text { Significant } P<.001-\text { ranked } 2 / 4 \\
\text { Significant } P<.001-\text { ranked } 1 / 4 \\
\text { Relevant (determined by literature review, doctors and patients) - ranked } 1-5 / 17 \\
\text { Relevant (determined by patients' interviews) - ranked } 1 / 9\end{array}$ & $\begin{array}{l}\text { Six different treatments } \\
\text { Dx, exercise, manual therapy, acupuncture } \\
\text { Exercise } \\
\text { Acupuncture, infrared treatment (minor, moderate, major reduction) } \\
\text { Surgical vs. non-surgical (same, less, none) } \\
\text { Surgical vs. non-surgical } \\
\text { HDS or home exercise, spinal manipulation }\end{array}$ & $\begin{array}{l}\text { Dima et al } / 2013 \\
\text { Dima et al } / 2015 \\
\text { François et al/2018 } \\
\text { Chen et al/2015 } \\
\text { Klojgaard et al/2014 } \\
\text { Klojgaard et al/2012 } \\
\text { Haanstra et al/2013 }\end{array}$ \\
\hline $\begin{array}{l}\text { Capacity to realize common/ } \\
\text { leisure/daylife activities }\end{array}$ & $\begin{array}{l}\text { Relevant (determined by patients) }- \text { ranked in top } 3 \\
\text { Significant } P<.001 \text { (positive) - ranked } 2 / 4 \\
\text { Relevant (determined by patients' interviews) - ranked 2/9 } \\
\text { Relevant (determined by literature review, doctors and patients) - ranked 1-5/17 }\end{array}$ & $\begin{array}{l}\text { Rehabilitation program + exercise } \\
\text { Surgical vs. non-surgical (same, fewer, none) } \\
\text { HDS or home exercise, spinal manipulation surgical vs. non-surgical }\end{array}$ & $\begin{array}{l}\text { Verbrugghe et al/2016 } \\
\text { Klojgaard et al/2014 } \\
\text { Haanstra et al/2013 } \\
\text { Klojgaard et al/2012 }\end{array}$ \\
\hline $\begin{array}{l}\text { Fit to patients' life/ } \\
\text { convenience }\end{array}$ & $\begin{array}{l}\text { Relevant (determined during focus group) same weight, top } 4 \\
\text { Relevant (determined during focus group) same weight, top } 4 \\
\text { Relevant (validated questionnaire) most important according to authors - ranked } 1 / 4 \\
\text { Relevant (determined during focus group) time management and flexible time-tables for } 18 / 18 \text { persons, } \\
\text { fit to patients' capacities for } 18 / 18 \text { persons }\end{array}$ & $\begin{array}{l}\text { Six different treatments } \\
\text { Dx, exercise, manual therapy, acupuncture } \\
\text { Exercise } \\
\text { Physical exercises program }\end{array}$ & $\begin{array}{l}\text { Dima et al/2013 } \\
\text { Dima et al/2015 } \\
\text { François et al/2018 } \\
\text { Slade et al/2009 }\end{array}$ \\
\hline $\begin{array}{l}\text { Frame/design of the } \\
\text { treatment (supervision or not } \\
\text { and individual or group) }\end{array}$ & $\begin{array}{l}\text { Significant } P<.001 \text { for group with supervision }- \text { attribute ranked } 4 / 6-\text { weight } 17 \% \\
\text { Relevant (determined during focus group) Non-clinical setting for } 16 / 18 \text { persons, close supervision for } \\
16 / 18 \text { persons and in group for } 11 / 18 \text { persons } \\
\text { Significant } P<.01 \text { preference for small group }- \text { ranked } 1 / 5 \\
\text { Relevant (determined by patients' interviews) } 9 / 9\end{array}$ & $\begin{array}{l}\text { Exercise (Individual w/o supervision, group w/o supervision) } \\
\text { Physical exercises program } \\
\text { Pain management program (individual, 2-6, 7-12, more than 12) } \\
\text { HDS or home exercise, spinal manipulation }\end{array}$ & $\begin{array}{l}\text { Aboagye et al/2017 } \\
\text { Slade et al/2009 } \\
\text { Yi et al/2011 } \\
\text { Haanstra et al/2013 }\end{array}$ \\
\hline $\begin{array}{l}\text { Providers' attitudes and } \\
\text { characteristics }\end{array}$ & $\begin{array}{l}\text { Relevant (determined by patients' interviews) - ranked } 9 / 9 \\
\text { Relevant (determined during focus group) encouraging instructors and their quality teaching skills, take } \\
\text { time to listen and shared decision-making for } 18 / 18 \text { persons } \\
\text { Relevant (determined by focus group) conscientious, knowledgeable, empathic, respectful and } \\
\text { trustworthy, outside the top } 4 \\
\text { Non-significant - ranked } 3 / 5\end{array}$ & $\begin{array}{l}\text { HDS or home exercise, spinal manipulation physical exercises program } \\
\text { Six different treatments } \\
\text { Pain management program (nurse, pharmacist, physiotherapist, GP, } \\
\text { psychologist, pain team) }\end{array}$ & $\begin{array}{l}\text { Haanstra et al/2013 } \\
\text { Slade et al } / 2009 \\
\text { Dima et al/2013 } \\
\text { Yi et al/2011 }\end{array}$ \\
\hline Credibility of treatment & $\begin{array}{l}\text { Relevant (determined during focus group) Same weight, top } 4 \\
\text { Relevant (determined during focus group) Same weight but prioritised by authors, top } 4 \\
\text { Relevant (determined by patients' interviews) Awareness and Confidence in treatment options - ranked } \\
1 / 11 \text { - weight } 16.2 \%\end{array}$ & $\begin{array}{l}\text { Six different treatments } \\
\text { Dx, exercise, manual therapy, acupuncture } \\
\text { CAM }\end{array}$ & $\begin{array}{l}\text { Dima et al/2013 } \\
\text { Dima et al/2015 } \\
\text { Hsu et al/2010 }\end{array}$ \\
\hline Capacity to return to work & $\begin{array}{l}\text { Relevant (determined by patients) - ranked } 2 / 5 \text { - weight } 14.29 \% \\
\text { Relevant (determined by patients) ranked in top } 3 \\
\text { Relevant (determined by literature review, doctors and patients) - ranked 6-17/17 }\end{array}$ & $\begin{array}{l}\text { Physiotherapy } \\
\text { Rehabilitation program + exercise } \\
\text { Surgical vs. non-surgical }\end{array}$ & $\begin{array}{l}\text { Gardner et al/2015 } \\
\text { Verbrugghe et al/2016 } \\
\text { Klojgaard et al/2012 }\end{array}$ \\
\hline Treatment frequency & $\begin{array}{l}\text { Significant } P<.001 \text { for Once or two times per week - attribute ranked } 3 / 6-\text { weight } 18 \% \\
\text { Significant } P<.01 \text { preference for fewer sessions over a longer period - ranked } 2 / 5 \\
\text { Relevant (determined by literature review, doctors and patients) - ranked } 6-17 / 17\end{array}$ & $\begin{array}{l}\text { Exercise (once, 2, } 3 \text { per week) } \\
\text { Pain management program (10, 5, 2, } 1 \text { sessions a week over 2, 4, 10, } \\
20 \text { weeks) } \\
\text { Surgical vs. non-surgical }\end{array}$ & $\begin{array}{l}\text { Aboagye et al/2017 } \\
\text { Yi et al/2011 } \\
\text { Klojgaard et al/2012 }\end{array}$ \\
\hline
\end{tabular}

204 International Journal of Health Policy and Management, 2021, 10(4), 201-210 
Table 2. Continued

\begin{tabular}{|c|c|c|c|}
\hline Attribute & Importance/Ranking & Treatment Modality (Levels) & Reference/Year \\
\hline Onset of treatment efficacy & $\begin{array}{l}\text { Significant } P<.001-\text { ranked } 4 / 4 \\
\text { Significant } P<.001 \text { (negative) }- \text { not ranked, used as reference } \\
\text { Relevant (determined by literature review, doctors and patients) - ranked 1-5/17 }\end{array}$ & $\begin{array}{l}\text { Acupuncture, infrared treatment ( } 2,4,8 \text { courses) } \\
\text { Surgical vs. non-surgical ( } 1,3,6,12 \text { months) } \\
\text { Surgical vs. non-surgical }\end{array}$ & $\begin{array}{l}\text { Chen et al/2015 } \\
\text { Klojgaard et al/2014 } \\
\text { Klojgaard et al/2012 }\end{array}$ \\
\hline Energy/ability to sleep & $\begin{array}{l}\text { Relevant (determined by patients) - ranked } 5 / 5 \text { - weight } 6.35 \% \\
\text { Relevant (determined by patients' interviews) - ranked } 8 / 11 \text { - weight } 2.4 \% \\
\text { Relevant (determined by literature review, doctors and patients) } 6-17 / 17\end{array}$ & $\begin{array}{l}\text { Physiotherapy } \\
\text { CAM } \\
\text { Surgical vs. non-surgical }\end{array}$ & $\begin{array}{l}\text { Gardner et al/2015 } \\
\text { Hsu et al/2010 } \\
\text { Klojgaard et al/2012 }\end{array}$ \\
\hline Realize physical activities & $\begin{array}{l}\text { Relevant (determined by patients) - ranked } 1 / 5 \text { - weight } 49.2 \% \\
\text { Relevant (determined by literature review, doctors and patients) - ranked 6-17/17 }\end{array}$ & $\begin{array}{l}\text { Physiotherapy } \\
\text { Surgical vs. non-surgical }\end{array}$ & $\begin{array}{l}\text { Gardner et al/2015 } \\
\text { Klojgaard et al/2012 }\end{array}$ \\
\hline Type of exercise & $\begin{array}{l}\text { Significant } P<.001 \text { for cardiovascular training }- \text { attribute ranked } 2 / 6 \text { - weight } 19 \% \\
\text { Relevant (determined during focus group) Fun and varied exercises for } 18 / 18 \text { persons, water-based for } \\
8 / 18\end{array}$ & $\begin{array}{l}\text { Exercise (cardiovascular, strength, mindfulness-based training) } \\
\text { Physical exercises program }\end{array}$ & $\begin{array}{l}\text { Aboagye et al/2017 } \\
\text { Slade et al/2009 }\end{array}$ \\
\hline Risk of relapse & $\begin{array}{l}\text { Significant } P<.001 \text { for } 30 \% \text { risk (negative) - ranked } 3 / 4 \\
\text { Relevant (determined by literature review, doctors and patients) - ranked 1-5/17 }\end{array}$ & $\begin{array}{l}\text { Surgical vs. non-surgical }(10 \%, 20 \%, 30 \%) \\
\text { Surgical vs. non-surgical }\end{array}$ & $\begin{array}{l}\text { Klojgaard et al/2014 } \\
\text { Klojgaard et al/2012 }\end{array}$ \\
\hline $\begin{array}{l}\text { Improvement in emotional } \\
\text { state }\end{array}$ & $\begin{array}{l}\text { Relevant (determined by patients' interviews) Emotional state ranked 3/11 - weight } 8.3 \% \text { - Well-being } \\
\text { ranked } 6 / 11 \text { - weight } 3.5 \% \\
\text { Relevant (determined by literature review, doctors and patients) - ranked 6-17/17 }\end{array}$ & $\begin{array}{l}\text { CAM } \\
\text { Surgical vs. non-surgical }\end{array}$ & $\begin{array}{l}\text { Hsu et al/2010 } \\
\text { Klojgaard et al/2012 }\end{array}$ \\
\hline To have a social life & $\begin{array}{l}\text { Relevant (determined by patients) - ranked } 4 / 5 \text { - weight } 6.35 \% \\
\text { Relevant (determined by literature review, doctors and patients) - ranked 6-17/17 }\end{array}$ & $\begin{array}{l}\text { Physiotherapy } \\
\text { Surgical vs. non-surgical }\end{array}$ & $\begin{array}{l}\text { Gardner et al/2015 } \\
\text { Klojgaard et al/2012 }\end{array}$ \\
\hline Out-of pocket cost & $\begin{array}{l}\text { Significant } P<.001-\text { not ranked, used as reference } \\
\text { Relevant (determined by focus group) for } 10 / 18 \text { persons }\end{array}$ & $\begin{array}{l}\text { Acupuncture, Infrared treatment }(120,600,1000 \mathrm{CNY} \text { per course) } \\
\text { Physical exercises program }\end{array}$ & $\begin{array}{l}\text { Chen et al/2015 } \\
\text { Slade et al/2009 }\end{array}$ \\
\hline Knowledge about their body & $\begin{array}{l}\text { Relevant (determined by patients' interviews) ranked } 4 / 11 \text { - weight } 7.6 \% \\
\text { Relevant (determined by focus group) for } 18 / 18 \text { persons }\end{array}$ & $\begin{array}{l}\text { CAM } \\
\text { Physical exercises program }\end{array}$ & $\begin{array}{l}\text { Hsu et al/2010 } \\
\text { Slade et } \mathrm{al} / 2009\end{array}$ \\
\hline $\begin{array}{l}\text { Knowledge about treatment } \\
\text { and disease }\end{array}$ & $\begin{array}{l}\text { Relevant (determined by patients' interviews) - ranked } 5 / 9 \\
\text { Relevant (determined by focus group) for } 18 / 18 \text { persons }\end{array}$ & HDS or home exercise, spinal manipulation physical exercises program & $\begin{array}{l}\text { Haanstra et al/2013 } \\
\text { Slade et al/2009 }\end{array}$ \\
\hline $\begin{array}{l}\text { Knowledge about etiology and } \\
\text { access to real diagnostic }\end{array}$ & $\begin{array}{l}\text { Relevant (determined by patients' interviews) - ranked 6/9 } \\
\text { Relevant (determined during focus group), outside the top } 4\end{array}$ & HDS or home exercise, spinal manipulation six different treatments & $\begin{array}{l}\text { Haanstra et al/2013 } \\
\text { Dima et al/2013 }\end{array}$ \\
\hline Self-management capacities & $\begin{array}{l}\text { Relevant (determined by patients' interviews) - ranked } 3 / 9 \\
\text { Relevant (determined by focus group), outside the top } 4\end{array}$ & HDS or home exercise, spinal manipulation six different treatments & $\begin{array}{l}\text { Haanstra et al/2013 } \\
\text { Dima et al/2013 }\end{array}$ \\
\hline
\end{tabular}




\section{Importance/Ranking}

Others symptoms non related Relevant (determined by researchers, doctors and patients) - ranked 6-17/17

to LBP Relevant (determined by patients' interviews) - ranked 7/11 - weight 2.7\%

Proximity

Non-significant - attribute ranked $6 / 6$ - weight $4 \%$

Significant $P<.01$ (negative) - ranked $4 / 5$

Significant $P<.001$ for none, exercise at work and wellness subsidies - attribute ranked 5/6 - weight

Incentives

Exercise intensity $17 \%$

Acceptability/logicality Significant $P<.001$ for High intensity - attribute ranked $1 / 6$ - weight 25\%

Suitability/appropriateness Relevant (validated questionnaire) same weight - ranked 2-4 over 4 attributes

Knowledge of the exercise Relevant (validated questionnaire) same weight - ranked 2-4 over 4 attributes Relevant (determined during focus group) for $18 / 18$ persons

Duration of efficacy $\quad$ Significant $P<.001-$ ranked $3 / 4$

Sensation of treatment $\quad$ Significant $P<.001-$ ranked $1 / 4$

Find motivation and seff-

confidence

Relevant (determined by patients' interviews) 8/9

Relevant (determined by patients' interviews) - ranked 4/9

Improvement biomechanica

functioning

Relaxation (mind and body)

Changes in way of thinking

Dramatic improvement in

overall health and well-being

Use of pain killers

Neurological deficits

Relevant (determined by patients' interviews) relaxation ranked 2/11 - weight $8.3 \%$ - mind-body-spirit ranked $10 / 11$ - weight $1.1 \%$ - mindfulness ranked $11 / 11$ - weight $0.5 \%$

Relevant (determined by patients' interviews) ranked 5/11 - weight 4.9\%

Relevant (determined by patients' interviews) ranked 9/11 - weight 1.5\%

Relevant (determined by literature review, doctors and patients) - ranked 6-17/17

Relevant (determined by literature review, doctors and patients) - ranked 6-17/17

Relevant (determined by patients) - ranked $3 / 5$ - weight $11.11 \%$

Coping skills

Seeking alternative treatment Relevant (determined by focus group), outside the top 4

\section{Treatment Modality (Levels)}

Surgical vs. non-surgical

CAM

Exercise (10, 20, 30 minutes)

(15,30,45,60,75,90,105,120 minutes from the clinic)

Exercise (none, wellness subsidies, exercise at work, discount coupon

Exercise (low, high, medium)

Exercise

Exercise

Physical exercises program

Acupuncture, Infrared treatment (2, 6, 12 months)

Reference/Year

Klojgaard et al/2012

Hsu et al/2010

Aboagye et al/2017

Yi et al/2011

Aboagye et al/2017

Aboagye et al/2017

François et al/2018

François et al/2018

Slade et al/2009

Chen et al/2015

Acupuncture, Infrared treatment (sore and numb, mild thermal and vibration)

HDS or Home exercise, Spinal manipulation

Chen et al/2015

Haanstra et al/2013

DS or Home exercise, Spinal manipulation

Haanstra et al/2013

CAM

Hsu et al $/ 2010$

CAM

Hsu et al $/ 2010$

CAM

Hsu et al/2010

Surgical vs. non-surgica

Klojgaard et al/2012

Surgical vs. non-surgical

Klojgaard et al/2012

Physiotherapy

Six different treatments

Gardner et al/2015

Dima et al/2013

ral practitioner; LBP, low back pain.

Difference between relevant and significant is related to the use of a statistical test or not. 
treatment efficacy, content of program, and energy/ability to sleep. Other attributes were only considered in one or two studies, thus making it difficult to identify which elements were really important for patients when choosing a treatment (see Table 2).

Some attributes provided conflicting results. This was particularly the case for the frame/design of the treatment and for the onset of treatment efficacy. While close supervision appeared to be valued by patients, the optimal size of the group supervised is still to be determined. In regard to the onset of treatment efficacy, patients seemed willing to wait a long time if the treatment would meet their expectations (ie, effectiveness).

Patients' preferences in term of treatment modality are reported in Table 3. One study did not compare treatments, ${ }^{49}$ considering only one treatment. Six studies only concerned the patients' preferences of attributes and not their treatment preferences. ${ }^{44-46,51-53}$ Consequently, only six studies investigated a specific preference for one of the treatments. ${ }^{41-43,47-48,50}$ Surgical treatment and acupuncture seemed to be less frequently preferred than other alternatives, such as physical exercise and medication. Most studies were about physical activities and compared various types of exercise, but no obvious tendency appeared.

\section{Discussion}

We identified which non-surgical treatments attributes for LBP were preferred by patients based on the scientific literature. As previously indicated, treatment preference is the option a patient chooses after considering the risks and benefits of the multiple options available for treatment of a clinical condition. ${ }^{11}$ In this setting, treatment preference was led by the preferences of patients according to the attributes and expected benefits, which are on their turn based on their experiences, knowledge and beliefs about the treatment. Previous authors have suggested that including patients' preferences in clinical decision-making about optimal treatment is a central aspect of practising evidence-based medicine. ${ }^{11,54-55}$ As such, to include patient preferences in the decision-making process has gained in importance among doctors. ${ }^{14}$ Knowing the patient's general expectations and preferences not only guides the choice of treatment, but may potentially improve the outcome of the treatment. ${ }^{56}$ Moreover, patients want to be included in this process, which leads to

Table 3. Preferences in Terms of Treatment Modality

\begin{tabular}{|c|c|c|}
\hline Author, Year & Treatment Modality & Preference \\
\hline $\begin{array}{l}\text { Francois } \\
\text { et al, } 2018\end{array}$ & SF training, MST & $\begin{array}{l}\text { SF training > MST } \\
\text { Preferences before the intervention: } 91.3 \% \text { preferred SF and } 8.7 \% \\
\text { MST. After the intervention: scores at } 3.88 \text { for SF and } 3.58 \text { for MST }\end{array}$ \\
\hline $\begin{array}{l}\text { Aboagye } \\
\text { et al, } 2017\end{array}$ & $\begin{array}{l}\text { Cardiovascular training, strength training, mindfulness- } \\
\text { based training }\end{array}$ & $\begin{array}{l}\text { Cardiovascular training }>\text { mindfulness-based training }>\text { strength } \\
\text { training } \\
\text { Significant at } P<.001\end{array}$ \\
\hline $\begin{array}{l}\text { Verbrugghe } \\
\text { et al, } 2017\end{array}$ & $\begin{array}{l}\text { Rehabilitation program (aerobe exercise therapy, posture } \\
\text { correction, breathing control, stabilization exercises and } \\
\text { home exercises) }\end{array}$ & $\begin{array}{l}\text { No precise preference. Household related activities were the most } \\
\text { preferred training activity }\end{array}$ \\
\hline $\begin{array}{l}\text { Chen } \\
\text { et al, } 2015\end{array}$ & Infrared therapy, acupuncture & $\begin{array}{l}\text { Infrared therapy }>\text { Acupuncture } \\
47.5 \% \text { choose infrared therapy against } 43.9 \% \text { who choose acupuncture }\end{array}$ \\
\hline $\begin{array}{l}\text { Dima } \\
\text { et al, } 2015\end{array}$ & Medication, exercise, manual therapy, acupuncture & $\begin{array}{l}\text { Exercise } \approx \text { Medication }>\text { Manual therapy }>\text { Acupuncture } \\
\text { Exercise } 3.64 \approx 3.63 \text { medications, manual therapy } 3.54 \text {, acupuncture } \\
3.25 \text {. } \\
\text { In a ranking exercise, } 152 \text { persons ranked medication first, whereas it } \\
\text { was } 88 \text { for exercise, } 89 \text { for manual therapy and } 24 \text { for acupuncture }\end{array}$ \\
\hline $\begin{array}{l}\text { Gardner } \\
\text { et al, } 2015\end{array}$ & Physiotherapy & No comparison with another treatment \\
\hline $\begin{array}{l}\text { Kløjgaard et al, } \\
2014\end{array}$ & Non-surgical and surgical interventions & $\begin{array}{l}\text { Non-surgical }>\text { Surgical interventions } \\
\text { Surgical interventions significant at } P<.001 \text { with negative preference }\end{array}$ \\
\hline $\begin{array}{l}\text { Dima } \\
\text { et al, } 2013\end{array}$ & $\begin{array}{l}\text { Medication, exercise, manual therapy, acupuncture, } \\
\text { combined and psychological approach, spinal fusion }\end{array}$ & No preference assessed \\
\hline $\begin{array}{l}\text { Haanstra et al, } \\
2013\end{array}$ & $\begin{array}{l}\text { High Dose Supervised Exercise, Home Exercise, Chiropractic } \\
\text { spinal manipulation }\end{array}$ & No preference assessed \\
\hline $\begin{array}{l}\text { Kløjgaard } \\
\text { et al, } 2012\end{array}$ & Non-surgical and surgical interventions & No preference assessed \\
\hline Yi et al, 2011 & $\begin{array}{l}\text { Pain management program (education, physical therapy, } \\
\text { coping with pain, medicines management) }\end{array}$ & $\begin{array}{l}\text { No precise preference. Patients seemed to be against Education and } \\
\text { Medicines Management when combined, significant at } P<.01 \text { with } \\
\text { negative preference }\end{array}$ \\
\hline $\begin{array}{l}\text { Slade } \\
\text { et al, } 2009\end{array}$ & Physical exercises program & $\begin{array}{l}\text { No precise preference. Some patients spontaneously cited water- } \\
\text { based exercise }(8 / 18)\end{array}$ \\
\hline
\end{tabular}

Abbreviations: SF, strength and flexibility; MST, motor skill training; CAMs, complementary and alternative medicines.

Note: When treatment $A$ is preferred to treatment $B$, we indicated $A>B$. 
greater satisfaction. ${ }^{57,58}$

According to this systematic review, the most frequently mentioned attributes in the preferences of patients for nonsurgical treatments were effectiveness, capacity to realize daily life activities, fit to the patient's life, providers' attitudes and characteristics, and the frame/design of the treatment (eg, supervised or not, in groups or individually). However, being mentioned does not guarantee that these attributes are considered important for patients. Indeed, these attributes are not of equal importance. By far, effectiveness is the attribute most mentioned (ie, 7 studies of 13) and the one that is frequently given the highest consideration by LBP patients. Other important attributes were capacities to realize daily life activities, fit to the patient's life, credibility of the treatment, capacity to return to work, and treatment frequency (ie, generally fewer sessions over a longer time period).

As per protocol, studies outside the scope of LBP were excluded from this systematic review. However, the results found are congruent with other chronic pain conditions..$^{35,37}$ To our knowledge, this study is the first systematic review on the topic of LBP patients' preferences for attributes of treatments underlying their choices. This study will be useful for future research in this field and especially for preparing new studies that aim to elicit the preferences of patients to offer them convenient healthcare services and to better fit the design of intervention toward LBP patients. Indeed, knowing the patient's preference for a given treatment is not sufficient to improve healthcare quality. This is why we need to know which attributes are important in the choice of a treatment modality by patients. This will help in clinical practice on how to adapt the design of treatments to better fit patients' preferences and incite patients to be more adherent. As an example, many studies have revealed that patients have preferences for home exercises, but have found that between $50 \%$ and $70 \%$ of chronic LBP patients did not perform these prescribed home exercises. ${ }^{19}$ As such, patients' preferences for specific attributes of home exercise could potentially impact clinical outcomes through adherence.

Several limits rise from this systematic mixed studies review. First, all included studies did not determine patients' preferences using the same method: a number were identified with focus groups, some with interviews and/ or questionnaires, and others with DCEs using different attributes and levels. In addition, some studies used statistical tests to compare the attributes, while others studies simply considered the attributes given spontaneously by patients or asked patients to perform a ranking. This could be interpreted as a methodological limitation for this review and could impede the comparability between results. Second, not all studies used the same attributes, which makes the comparison of attributes between studies even harder. Third, we indirectly assessed the risk of bias of the included studies using the MMAT which is imperfect considering that this tool mostly evaluates the quality of mixed-methods studies. However, we are not aware of specific tools to assess the risk of bias in preference studies. Fourth, all reviews, including the present one, is limited by the search strategy and the selection of databases, which may have led to some missed studies. Fifth, preferences may vary across populations with disparate demographic characteristics, but due to limited data provided in the studies we were not able to assess if these characteristics have an impact on patients' preferences. Sixth, some information is missing or insufficiently described in the studies retrieved, such as at what time in the consultation process the patients were asked for their preferences, the information they may have received about treatments, and data to determine if patients were comparable from one study to another. This information would have been helpful to better understand patients' preferences. Seventh, we attempted to report the attributes by the main treatment modalities (eg, exercise, acupuncture, surgical vs. non-surgical), but no specific pattern was found. A potential explanation for this is that each modality, even in the same category, can differ greatly from each other. Finally, included studies had various objectives, which may have led to different rankings or even omitting certain attributes. Despite the fact that we conducted a rigorous selection process in this systematic review, all these points are strong limitations that preclude establishing a clear ranking as to patients' preferences.

However, as said above, a strength of this review is that we followed a standard and rigorous method, thus allowing to find some key preferences in treatment attributes. Moreover, this review is in line with various international recommendations to consider patients' views in order to improve patientcentered care. $^{59}$ Although including patients in clinical decisions may be challenging, patient involvement may potentially have a significant effect on treatment outcomes. ${ }^{60}$ The benefits of patient involvement and the skills required to achieve this is thus a central aspect of practicing evidencebased medicine. ${ }^{60}$ In this sense, the present study is important as it aims to highlight patients' treatment preferences, which is pertinent for caregivers to know.

\section{Conclusion}

In this systematic mixed studies review, we found that effectiveness (ie, pain reduction) was the most important attribute considered by patients in their choice of a treatment. This attribute was cited in seven of the thirteen included studies and was systematically ranked first or second. Other important attributes were the capacity to realize daily life activities, fit to the patient's life, and credibility of the treatment, among others. However, these are not the only traits and future research is needed to clearly determine their relative importance. This research is important considering that patients' preference is essential in the decision-making process, since it could influence adherence to treatment and clinical outcomes. This is part of a process whereby healthcare providers should share treatment decisions with patients by listening to them, trying to understand them, and considering their wishes. ${ }^{50}$

\section{Acknowledgement}

We acknowledge the UETMISSS team at the CIUSSS de l'Estrie - CHUS. TGP is member of the FRQS-funded Centre de recherche de l'IUSMM. 


\section{Ethical issues}

This article does not contain any studies with human participants performed by any of the authors.

\section{Competing interests}

Authors declare that they have no competing interests.

\section{Authors' contributions}

TGP and MB conceived and conducted the study. TGP wrote the manuscript and $\mathrm{MB}$ revised it critically.

\section{Authors' affiliations}

${ }^{1}$ School of Public Health, University of Montreal, Montreal, QC, Canada. ${ }^{2}$ Research Center of the IUSMM, CIUSSS de l'Est de l'île de Montréal, Montreal, QC, Canada. ${ }^{3} \mathrm{CERDI}$, Université Clermont Auvergne, ClermontFerrand, France.

\section{Supplementary files}

Supplementary file 1 contains the complete search strategy based on keywords.

\section{References}

1. Schmidt CO, Raspe H, Pfingsten M, et al. Back pain in the German adult population: prevalence, severity, and sociodemographic correlates in a multiregional survey. Spine (Phila Pa 1976). 2007;32(18):20052011. doi:10.1097/BRS.0b013e318133fad8

2. Cassidy JD, Carroll LJ, Côté P. The Saskatchewan health and back pain survey. The prevalence of low back pain and related disability in Saskatchewan adults. Spine (Phila Pa 1976). 1998;23(17):1860-1866. doi:10.1097/00007632-199809010-00012

3. Hartvigsen J, Hancock MJ, Kongsted A, et al. What low back pain is and why we need to pay attention. Lancet. 2018;391(10137):23562367. doi:10.1016/s0140-6736(18)30480-x

4. National Institute of Neurological Disorders and Stroke. Low Back Pain Fact Sheet. https://www.ninds.nih.gov/Disorders/Patient-CaregiverEducation/Fact-Sheets/Low-Back-Pain-Fact-Sheet.

5. World Health Organization (WHO). Priority Medicines for Europe and the World. WHO; 2013.

6. Fayad F, Lefevre-Colau MM, Poiraudeau S, et al. Chronicity, recurrence, and return to work in low back pain: common prognostic factors. Ann Readapt Med Phys. 2004;47(4):179-189. doi:10.1016/j. annrmp.2004.01.005

7. Poiraudeau S, Lefevre-Colau MM, Fayad F, et al. Low back pain. EMC Rhumatol Orthop. 2004;1:295-319.

8. Véron O, Tcherniatinsky E, Fayad F, Revel M, Poiraudeau S. Chronic low back pain and functional restoring program: applicability of the Patient Acceptable Symptom State. Ann Readapt Med Phys. 2008; 51(8):642-649. doi:10.1016/j.annrmp.2008.08.003

9. Waddell G. Simple low back pain: rest or active exercise? Ann Rheum Dis. 1993;52(5):317-319. doi:10.1136/ard.52.5.317

10. Náfrádi L, Nakamoto K, Schulz PJ. Is patient empowerment the key to promote adherence? A systematic review of the relationship between self-efficacy, health locus of control and medication adherence. PLoS One. 2017;12(10):e0186458. doi:10.1371/journal.pone.0186458

11. Bowling A, Ebrahim S. Measuring patients' preferences for treatment and perceptions of risk. Qual Health Care. 2001;10 Suppl 1:i2-8.

12. Haldeman S, Dagenais S. A supermarket approach to the evidenceinformed management of chronic low back pain. Spine J. 2008;8(1):17. doi:10.1016/j.spinee.2007.10.009

13. Balagué F, Dudler J. An overview of conservative treatment for lower back pain. Int J Clin Rheumtol. 2011;6(3):281-290.

14. Aboagye E. Valuing individuals' preferences and health choices of physical exercise. Pain Ther. 2017;6(1):85-91. doi:10.1007/s40122017-0067-4

15. Leventhal $\mathrm{H}$, Brissette I, Leventhal EA. The common-sense model of self-regulation of health and illness. In: Cameron LD, Leventhal $\mathrm{H}$, eds. The Self-Regulation of Health and IIIness Behaviour. Routledge; 2003:42-65

16. The National Institute for Health and Care Excellence (NICE). Low Back Pain and Sciatica in Over 16s: Assessment and Management. London: NICE; 2016.

17. Institut national d'excellence en santé et en services sociaux (INESSS). Les normes de production des revues systématiques.
Guide méthodologique. Montréal: INESSS; 2013:44.

18. Pluye P, Robert E, Cargo M, et al. Proposal: A Mixed Methods Appraisal Tool for Systematic Mixed Studies Reviews. Montreal: McGill Univ; 2011:1-8.

19. Beinart NA, Goodchild CE, Weinman JA, Ayis S, Godfrey EL. Individual and intervention-related factors associated with adherence to home exercise in chronic low back pain: a systematic review. Spine J. 2013;13(12):1940-1950. doi:10.1016/j.spinee.2013.08.027

20. Finch AP, Dritsaki M, Jommi C. Generic preference-based measures for low back pain: which of them should be used? Spine (Phila Pa 1976). 2016;41(6):E364-374. doi:10.1097/brs.0000000000001247

21. Slade SC, Patel S, Underwood M, Keating JL. What are patient beliefs and perceptions about exercise for nonspecific chronic low back pain? a systematic review of qualitative studies. Clin J Pain. 2014;30(11):9951005. doi:10.1097/ajp.0000000000000044

22. Bishop MD, Bialosky JE, Penza CW, Beneciuk JM, Alappattu MJ. The influence of clinical equipoise and patient preferences on outcomes of conservative manual interventions for spinal pain: an experimental study. J Pain Res. 2017;10:965-972. doi:10.2147/jpr.s130931

23. NICE guidelines for low-back pain. J Natl Med Assoc. 2009;101:974 doi:10.1016/S0027-9684(15)31051-8

24. George SZ, Robinson ME. Preference, expectation, and satisfaction in a clinical trial of behavioral interventions for acute and subacute low back pain. J Pain. 2010;11(11):1074-1082. doi:10.1016/j. jpain.2010.02.016

25. Arefyev A, Lechauve JB, Gay C, et al. Mobile application development through qualitative research in education program for chronic low back patients. Ann Phys Rehabil Med. 2017;60:e102-e103. doi:10.1016/j. rehab.2017.07.070

26. Perrot S, Concas V, Allaert F, Laroche F. Deciding on analgesic prescription dosing for acute back pain: once daily or more? Presse Med. 2008;37(1 Pt 1):14-20. doi:10.1016/j.lpm.2007.09.011

27. Blanchette MA, Rivard M, Dionne CE, Hogg-Johnson S, Steenstra I. Workers' characteristics associated with the type of healthcare provider first seen for occupational back pain. BMC Musculoskelet Disord. 2016;17(1):428. doi:10.1186/s12891-016-1298-y

28. Cooper K, Smith BH, Hancock E. Patients' perceptions of selfmanagement of chronic low back pain: evidence for enhancing patient education and support. Physiotherapy. 2009;95(1):43-50. doi:10.1016/j.physio.2008.08.005

29. Lynch AD, Bove AM, Ammendolia C, Schneider M. Individuals with lumbar spinal stenosis seek education and care focused on selfmanagement-results of focus groups among participants enrolled in a randomized controlled trial. Spine J. 2018;18(8):1303-1312. doi:10.1016/j.spinee.2017.11.019

30. Pauwels C, Roren A, Gautier A, et al. Home-based cycling program tailored to older people with lumbar spinal stenosis: barriers and facilitators. Ann Phys Rehabil Med. 2018;61(3):144-150. doi:10.1016/j. rehab.2018.02.005

31. Zandwijk $\mathrm{P}$, van Koppen B, van Mameren $\mathrm{H}$, Winkens $\mathrm{B}$, de Bie R. Physical activity preferences of patients experiencing non-specific low back pain. Eur J Physiother. 2018;20(1):51-57. doi:10.1080/2167916 9.2017.1357753

32. Liddle SD, Baxter GD, Gracey JH. Chronic low back pain: patients' experiences, opinions and expectations for clinical management. Disabil Rehabil. 2007;29(24):1899-1909. doi:10.1080/09638280701189895

33. Iles RA, Taylor NF, Davidson M, O'Halloran PD. Patient recovery expectations in non-chronic non-specific low back pain: a qualitative investigation. J Rehabil Med. 2012;44(9):781-787. doi:10.2340/16501977-1019

34. Bishop MD, Bialosky JE, Cleland JA. Patient expectations of benefit from common interventions for low back pain and effects on outcome: secondary analysis of a clinical trial of manual therapy interventions. J Man Manip Ther. 2011;19(1):20-25. doi:10.1179/10669811 0x12804993426929

35. Maiers M, Hondras MA, Salsbury SA, Bronfort G, Evans R. What do patients value about spinal manipulation and home exercise for backrelated leg pain? A qualitative study within a controlled clinical trial. Man Ther. 2016;26:183-191. doi:10.1016/j.math.2016.09.008

36. Takeda O, Chiba D, Ishibashi Y, Tsuda E. Patient-physician differences in desired characteristics of NSAID plasters: an online survey. Pain Res Manag. 2017;2017:5787854. doi:10.1155/2017/5787854 
37. Chancellor J, Martin M, Liedgens H, Baker MG, Müller-Schwefe $\mathrm{GH}$. Stated preferences of physicians and chronic pain sufferers in the use of classic strong opioids. Value Health. 2012;15(1):106-117. doi:10.1016/j.jval.2011.07.002

38. Bethge M. Patient preferences and willingness to wait for a workrelated orthopaedic rehabilitation: a discrete choice experiment. Gesundheitswesen. 2009;71(3):152-160.

39. Kløjgaard ME, Hess S. Understanding the formation and influence of attitudes in patients' treatment choices for lower back pain: testing the benefits of a hybrid choice model approach. Soc Sci Med. 2014; 114:138-150. doi:10.1016/j.socscimed.2014.05.058

40. Slade SC, Keating JL. Effects of preferred-exercise prescription compared to usual exercise prescription on outcomes for people with non-specific low back pain: a randomized controlled trial [ACTRN12608000524392]. BMC Musculoskelet Disord. 2009;10:14. doi:10.1186/1471-2474-10-14

41. Francois SJ, Lanier VM, Marich AV, Wallendorf M, Van Dillen LR. A cross-sectional study assessing treatment preference of people with chronic low back pain. Arch Phys Med Rehabil. 2018;99(12):24962503. doi:10.1016/j.apmr.2018.04.027

42. Aboagye E, Hagberg J, Axén I, et al. Individual preferences for physical exerciseas secondary prevention for non-specificlow back pain:Adiscrete choice experiment. PLoS One. 2017;12(12):e0187709. doi:10.1371/ journal.pone.0187709

43. Yi D, Ryan M, Campbell S, et al. Using discrete choice experiments to inform randomised controlled trials: an application to chronic low back pain management in primary care. Eur J Pain. 2011;15(5):531.e531510. doi:10.1016/j.ejpain.2010.10.008

44. Hsu C, Bluespruce J, Sherman K, Cherkin D. Unanticipated benefits of CAM therapies for back pain: an exploration of patient experiences. J Altern Complement Med. 2010;16(2):157-163. doi:10.1089/ acm.2009.0188

45. Slade SC, Molloy E, Keating JL. People with non-specific chronic low back pain who have participated in exercise programs have preferences about exercise: a qualitative study. Aust J Physiother. 2009;55(2):115-121. doi:10.1016/s0004-9514(09)70041-8

46. Verbrugghe J, Haesen M, Spierings R, et al. Skill training preferences and technology use in persons with neck and low back pain. Disabil Rehabil Assist Technol. 2017;12(8):801-807. doi:10.1080/17483107.2 016.1269208

47. Chen LC, Cheng LJ, Zhang Y, He X, Knaggs RD. Acupuncture or low frequency infrared treatment for low back pain in Chinese patients: a discrete choice experiment. PLoS One. 2015;10(5):e0126912. doi:10.1371/journal.pone.0126912
48. Dima A, Lewith GT, Little P, et al. Patients' treatment beliefs in low back pain: development and validation of a questionnaire in primary care. Pain. 2015;156(8):1489-1500. doi:10.1097/j.pain.0000000000000193

49. Gardner T, Refshauge K, McAuley J, Goodall S, Hübscher M, Smith L. Patient led goal setting in chronic low back pain-What goals are important to the patient and are they aligned to what we measure? Patient Educ Couns. 2015;98(8):1035-1038. doi:10.1016/j. pec.2015.04.012

50. Kløjgaard ME, Manniche C, Pedersen LB, Bech M, Søgaard R. Patient preferences for treatment of low back pain-a discrete choice experiment. Value Health. 2014;17(4):390-396. doi:10.1016/j. jval.2014.01.005

51. Dima A, Lewith GT, Little P, Moss-Morris R, Foster NE, Bishop FL. Identifying patients' beliefs about treatments for chronic low back pain in primary care: a focus group study. Br J Gen Pract. 2013;63(612):e490498. doi:10.3399/bjgp13X669211

52. Haanstra TM, Hanson L, Evans R, et al. How do low back pain patients conceptualize their expectations regarding treatment? Content analysis of interviews. Eur Spine J. 2013;22(9):1986-1995. doi:10.1007/s00586-013-2803-8

53. Kløjgaard ME, Bech M, Søgaard R. Designing a stated choice experiment: the value of a qualitative process. J Choice Model. 2012;5(2):1-18. doi:10.1016/S1755-5345(13)70050-2

54. Bowling A, Rowe G, Lambert N, et al. The measurement of patients' expectations for health care: a review and psychometric testing of a measure of patients' expectations. Health Technol Assess. 2012;16(30):i-xii, 1-509. doi:10.3310/hta16300

55. Poder TG. Editorial: better understanding health preferences. Int J Health Prefer Res. 2016;1:1. doi:10.21965/IJHPR.2016.E

56. Mondloch MV, Cole DC, Frank JW. Does how you do depend on how you think you'll do? a systematic review of the evidence for a relation between patients' recovery expectations and health outcomes. CMAJ. 2001;165(2):174-179.

57. Strull WM, Lo B, Charles G. Do patients want to participate in medical decision making? JAMA. 1984;252(21):2990-2994.

58. Main CJ, Foster N, Buchbinder R. How important are back pain beliefs and expectations for satisfactory recovery from back pain? Best Pract Res Clin Rheumatol. 2010;24(2):205-217. doi:10.1016/j. berh.2009.12.012

59. Greene SM, Tuzzio L, Cherkin D. A framework for making patientcentered care front and center. Perm J. 2012;16(3):49-53.

60. Elwyn G, Frosch D, Thomson R, et al. Shared decision making: a model for clinical practice. J Gen Intern Med. 2012;27(10):1361-1367. doi:10.1007/s11606-012-2077-6 